\title{
Control of a Reduced Size Model of US Navy Crane Using Only Motor Position Sensors`
}

\author{
Bálint Kiss ${ }^{1}$, Jean Lévine ${ }^{1}$, and Philippe Mullhaupt ${ }^{1}$ \\ Centre Automatique et Systèmes, École des Mines de Paris, 35, rue Saint-Honoré \\ F-77305 Fontainebleau, France, E-mail:\{kiss,levine,mulhaupt\}@cas.ensmp.fr
}

\begin{abstract}
Two control problems related to a particular underactuated mechanical system, the reduced size US Navy crane, are addressed. The open-loop motion planning problem is solved by showing that the model of the crane is differentially flat with a flat output comprising the coordinates of the load as its first components. The closed-loop global asymptotic stabilization of equilibria is achieved using an output feedback regulator of proportional-derivative type. The extension of this approach to tracking is analyzed based on simulation results.
\end{abstract}

\section{Introduction}

Cranes constitute good examples of nonlinear oscillating pendulum- like systems with challenging industrial applications. Their control has been approached by various techniques, linear $[1,7,8,15]$ and nonlinear $[6,9,14]$.

Cranes present two interesting properties from the control engineering point of view. They are underactuated, i.e. the number of actuators is less than the number of degrees of freedom (see [2]). Moreover, only partial information can be used for closed-loop control, i.e. measurement of the whole state is unavailable (especially as far as the rope angles or the load position are concerned) (see [13]).

In this paper the particular example of the reduced size US Navy crane is studied. (All presented methods can be extended to a large class of similar equipment [10].)

Two control problems are addressed: open-loop real-time motion planning and closed-loop stabilization. The solution presented to the open-loop motion planning problem allows to calculate the necessary control input as to move the load along any (sufficiently smooth) trajectory in the working space using the flatness property $[3,4,5]$ of the system. The second control problem is the closed-loop stabilization of both an equilibrium and a trajectory. Since the only measurements available are the motor positions (recall that the load position or the rope angles are not measured) this problem can not be solved using full state feedback. Instead, a classical PD output regulation

\footnotetext{
^ Research supported by the Nonlinear Control Network, European Commission's Training and Mobility of Researchers (TMR) Contract \# ERBFMRX-CT970137 http://www.supelec.fr/lss/NCN/crane.html\#PDP
} 
is proposed. Global closed-loop stability of equilibria can be proved using LaSalle's invariance principle [12] and the particular structure of the crane dynamics. Note that this result is of particular practical interest to reduce the time to damp the oscillations of the load during harbor operations [16].

Simulation results show that the same regulator may also be used for tracking. Based on experimental considerations, it appears that our PD regulator together with flatness based trajectory planning outperforms the globally stabilizing regulator, though no proof is presented herein.

The remaining part of the paper is organized as follows. The next section is devoted to the general description of the experimental setup. Modeling equations are given in Section 3. The solution of the open-loop motion planning problem is presented in Section 4 based on the flatness property of the model. Asymptotic global stability of equilibria in closed-loop using output feedback regulators of proportional-derivative type is studied in Section 5. Simulation results of an extension of the same controller with open-loop trajectory planning for tracking are presented in Section 6.

\section{General description of the experimental setup}

The reduced scale (1:80 size) model $^{1}$ of the US-Navy crane is depicted in Figure 1. Four DC motors (three of them winching ropes) are mounted on the structure allowing to manipulate the load in a three dimensional workspace.

The control objective is to move the load swiftly from an initial position to a desired final position without sway and avoiding obstacles. Since the accelerations of the motors tend to create oscillations of the load, simultaneously fast and swayless displacements are hard to realize.

The reduced size model comprises:

- A load (maximal nominal mass: 800g)

- A mobile pulley guiding the rope which hoists the load.

- A rotate platform actuated by the DC motor no.4 using a synchronous belt transmission.

- A hoisting system mounted on the rotate platform comprising three ropes winched by three DC motors (motors no.1,2,3):

- A horizontal rope attached to the mobile pulley and ending up on the winch of motor 1 .

- a vertical rope attached to the mobile pulley and ending up on the winch of motor 2 . This rope prevents the mobile pulley from falling.

- A rope attached to the load passing through the mobile pulley and ending up on the winch of motor 3.

- A power electronics unit ${ }^{2}$. It receives sensor signals from the incremental encoders mounted on the motor axes and transmits them to a computer. It also provides the necessary power amplification to the DC motors.

\footnotetext{
${ }^{1}$ the reduced scale model was made by Walter Rumsey, Paris, France

${ }^{2}$ the power electronics unit was made by the Institut d'Automatique of the École Polytechnique Fédérale de Lausanne, Switzerland
} 


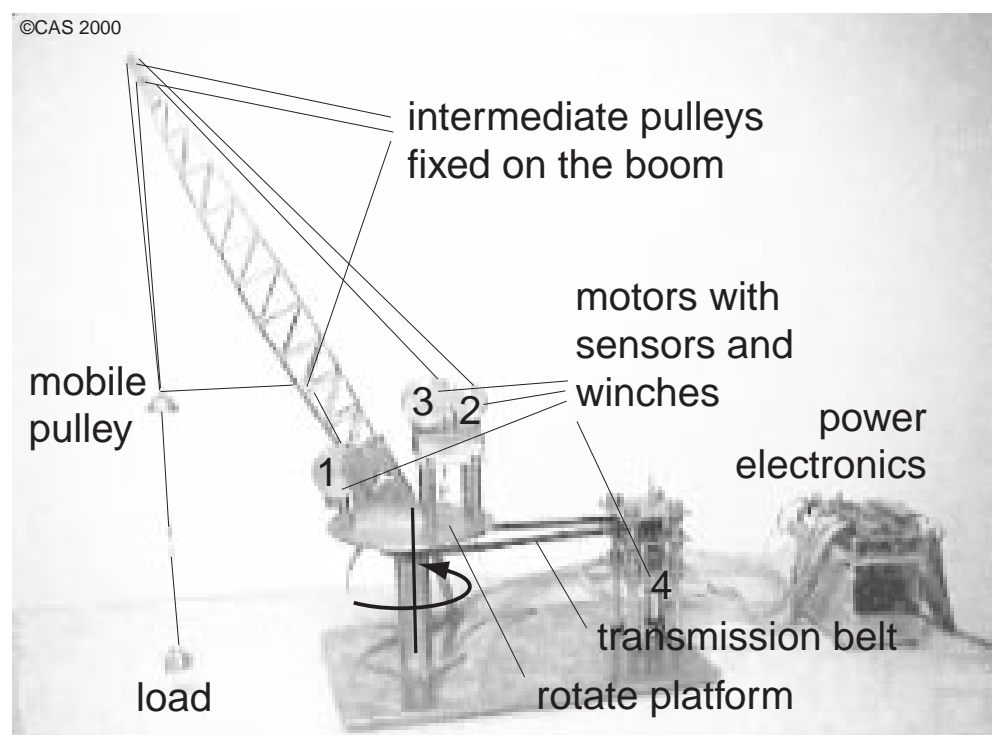

Fig. 1. The reduced (1:80) size US Navy crane in the authors' lab.

The control algorithm is implemented on a personal computer equipped with a standard data acquisition card. The measured signals are the rotation angles of the motor axes which allow to calculate directly the rope lengths and the rotation angle of the platform. The corresponding velocities are calculated using numerical derivation.

\section{Model Equations}

Figure 2 gives the schematic representation of the crane. The electronic time constants are negligible w.r.t. the mechanical time constants. Consequently, the input variables of the model are the torques $T_{1}, T_{2}, T_{3}, T_{4}$ delivered by the motors no. $1-4$ respectively.

Observe that along each rope there is an intermediate pulley fixed to the boom. Since the length of the rope sections between these pulleys and the winches of the motors are constant, we consider that the motors drive directly the axis of the intermediate pulleys and we reduce all rotating inertias along each rope on these axes. The same simplification is made concerning the belt transmission, i.e. we consider that motor 4 drives directly the axis of the rotate platform and we reduce all rotating inertias to this axis. All ropes are considered to be rigid.

The following variables and inertia parameters are introduced: 


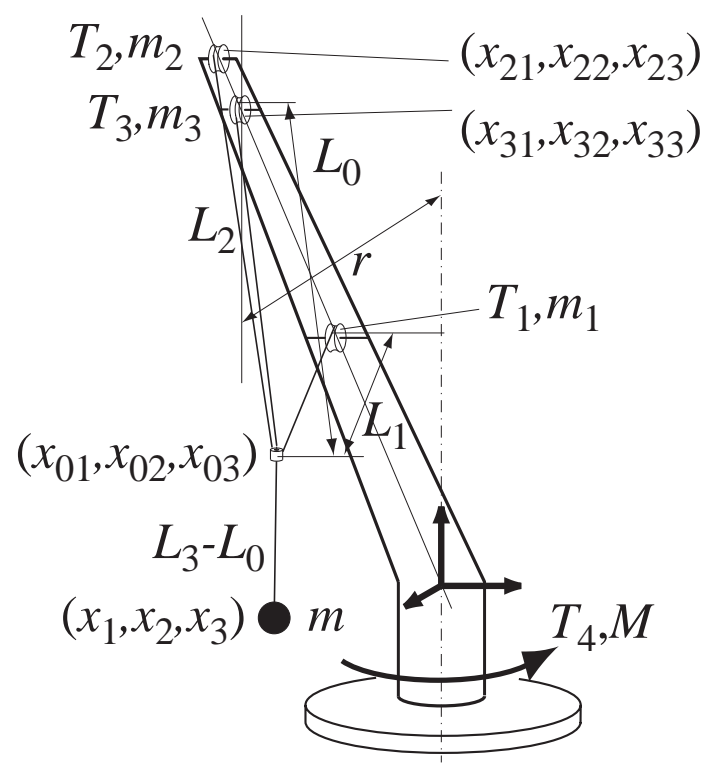

Fig. 2. Simplified representation of the 3D US Navy crane

- $x_{1}, x_{2}, x_{3}$ : position of the load,

- $x_{01}, x_{02}, x_{03}$ : position of the mobile pulley,

- $x_{11}, x_{12}, x_{13}$ : position of the pulley winding the horizontal rope attached to the mobile pulley,

- $x_{21}, x_{22}, x_{23}$ : position of the pulley winding the vertical rope attached to the mobile pulley,

- $x_{31}, x_{32}, x_{33}$ : position of the pulley hoisting the load,

- $L_{0}$ : length of the rope section between the pulley hoisting the load and the mobile pulley,

- $L_{1}$ : length of the horizontal rope attached to the mobile pulley,

- $L_{2}$ : length of the vertical rope attached to the mobile pulley,

- $L_{3}$ : length of the rope attached to the load,

- $m$ : mass of the load,

- $m_{0}$ : mass of the mobile pulley,

- $m_{1}, m_{2}, m_{3}$ : rotating inertias reduced to the intermediate pulleys' axis,

- $M$ : rotating inertia reduced to the axis of the rotate platform

The construction of the crane is such that the three intermediate pulleys are aligned. Thus we introduce two geometric parameters $\alpha_{2}$ and $\alpha_{3}$ such that $x_{i j}=\alpha_{i} x_{1 j}$ for $=2,3$ and $j=1,2,3$. Observe also that $x_{i 3}=$ constant, $i=1,2,3$.

Denote by $q=\left(q_{1} \ldots q_{12}\right)^{T}=\left(x_{1}, x_{2}, x_{3}, x_{01}, x_{02}, x_{03}, x_{11}, x_{12}, L_{0}, L_{1}\right.$, $\left.L_{2}, L_{3}\right)^{T}$ the vector of system variables. 
Geometric constraints are present due to the various cable distances that need to be compatible with each other.

Theorem 1. The dynamics associated to the US Navy crane are

$$
\begin{aligned}
m \ddot{x}_{1} & =\lambda_{1}\left(x_{1}-x_{01}\right) \\
m \ddot{x}_{2} & =\lambda_{1}\left(x_{2}-x_{02}\right) \\
m \ddot{x}_{3} & =\lambda_{1}\left(x_{3}-x_{03}\right)-m g \\
m_{0} \ddot{x}_{01} & =-\lambda_{1}\left(x_{1}-x_{01}\right)-\lambda_{2}\left(x_{01}-x_{11}\right)-\lambda_{3}\left(x_{01}-\alpha_{2} x_{11}\right)-\lambda_{4}\left(x_{01}-\alpha_{3} x_{11}\right) \\
m_{0} \ddot{x}_{02} & =-\lambda_{1}\left(x_{2}-x_{02}\right)-\lambda_{2}\left(x_{02}-x_{12}\right)-\lambda_{3}\left(x_{02}-\alpha_{2} x_{12}\right)-\lambda_{4}\left(x_{02}-\alpha_{3} x_{12}\right) \\
m_{0} \ddot{x}_{03} & =-\lambda_{1}\left(x_{3}-x_{03}\right)-\lambda_{2}\left(x_{03}-x_{13}\right)-\lambda_{3}\left(x_{03}-\alpha_{2} x_{13}\right)-\lambda_{4}\left(x_{03}-\alpha_{3} x_{13}\right)-m_{0} g \\
0 & =\lambda_{1}\left(L_{3}-L_{0}\right)-\lambda_{4} L_{0} \\
m_{1} \ddot{L}_{1} & =-\lambda_{2} L_{1}+T_{1} \\
m_{2} \ddot{L}_{2} & =-\lambda_{3} L_{2}+T_{2} \\
m_{3} \ddot{L}_{3} & =-\lambda_{1}\left(L_{3}-L_{0}\right)+T_{3} \\
M \ddot{x}_{11} & =-\lambda_{2}\left(x_{01}-x_{11}\right)-\alpha_{2} \lambda_{3}\left(x_{01}-\alpha_{2} x_{11}\right)-\alpha_{3} \lambda_{4}\left(x_{01}-\alpha_{3} x_{11}\right)-\lambda_{5} x_{11}-T_{4} x_{12} \\
M \ddot{x}_{12} & =-\lambda_{2}\left(x_{02}-x_{12}\right)-\alpha_{2} \lambda_{3}\left(x_{02}-\alpha_{2} x_{12}\right)-\alpha_{3} \lambda_{4}\left(x_{02}-\alpha_{3} x_{12}\right)-\lambda_{5} x_{12}+T_{4} x_{11}
\end{aligned}
$$

subject to the constraints

$$
\begin{aligned}
& C_{1}=\frac{1}{2}\left(\sum_{i=1}^{3}\left(x_{i}-x_{0 i}\right)^{2}-\left(L_{3}-L_{0}\right)^{2}\right)=0 \\
& C_{2}=\frac{1}{2}\left(\sum_{i=1}^{3}\left(x_{0 i}-x_{1 i}\right)^{2}-L_{1}^{2}\right)=0 \\
& C_{3}=\frac{1}{2}\left(\sum_{i=1}^{3}\left(x_{0 i}-\alpha_{2} x_{1 i}\right)^{2}-L_{2}^{2}\right)=0 \\
& C_{4}=\frac{1}{2}\left(\sum_{i=1}^{3}\left(x_{0 i}-\alpha_{3} x_{1 i}\right)^{2}-L_{0}^{2}\right)=0 \\
& C_{5}=\frac{1}{2}\left(x_{31}^{2}+x_{32}^{2}-r^{2}\right)=0
\end{aligned}
$$

The multipliers $\lambda_{1} \ldots \lambda_{5}$ are associated to the constraints $C_{1} \ldots C_{5}$. Recall that the kinetic and the potential energy of the system read

$$
\begin{aligned}
& W_{k}=\frac{1}{2}\left(\sum_{i=1}^{3}\left(m \dot{x}_{i}^{2}+m_{0} \dot{x}_{0 i}^{2}\right)+\sum_{i=1}^{2} M \dot{x}_{1 i}^{2}+m_{1} \dot{L}_{1}^{2}+m_{2} \dot{L}_{2}^{2}+m_{3} \dot{L}_{3}^{2}\right) \\
& W_{p}=m g x_{3}+m_{0} g x_{03},
\end{aligned}
$$

hence the Lagrangian is defined by $\mathcal{L}=W_{k}-W_{p}$. A proof of this result can be found in [10]. 


\section{$4 \quad$ Flatness and Motion Planning}

\subsection{Flatness}

A flat output is given by $Y=\left(Y_{1}, \ldots, Y_{4}\right)^{T}=\left(x_{1}, x_{2}, x_{3}, x_{03}\right)^{T}$, the coordinates of the load and the height of the mobile pulley. As long as we omit freefall reference trajectories, i.e. $\ddot{x}_{3} \neq g$, the first three equations of (1) give $\lambda_{1}$, $x_{01}$ and $x_{02}$ as functions of $Y$ and $\ddot{Y}$. Equations 5 to 8 of (1) and Constraints $C_{1}, C_{4}$ and $C_{5}$ of (2) can then be used to express $\left\{\lambda_{2}, \lambda_{3}, \lambda_{4}, x_{11}, \lambda_{12}, L_{3}, L_{0}\right\}$ as expressions of $\lambda_{1}, x_{1}, x_{2}, x_{3}, x_{01}, x_{02}, x_{03}$ and derivatives up to order 2 (thus giving expressions involving $Y, \dot{Y}, \ddot{Y}, Y^{(3)}$ and $\left.Y^{(4)}\right)$. Next, the constraints $C_{2}$ and $C_{3}$ give $L_{1}$ and $L_{2}$. The other equations of (1) give the inputs $T_{1}, T_{2}$, $T_{3}$ and $T_{4}\left(T_{4}\right.$ is obtained after solving the last two equations for $T_{4}$ and $\left.\lambda_{5}\right)$. The inputs are expressions of $Y$ and its derivatives up to order 6 .

\subsection{Motion planning}

Assume that the position, velocity, acceleration, jerk and all derivatives up to 6 th order of the flat output (including the position of the load) at the start time $t_{I}$ are given by $\left(Y_{I}, \dot{Y}_{I}, \ddot{Y}_{I}, \ldots, Y_{I}^{(5)}, Y_{I}^{(6)}\right)$ and the desired final configuration of the flat output at time $t_{F}$ is $\left(Y_{F}, \dot{Y}_{F}, \ddot{Y}_{F}, \ldots, Y_{F}^{(5)}, Y_{F}^{(6)}\right)$. We can construct 13 th degree polynomials,

$$
Y_{c i}(t)=Y_{I i}+\left(Y_{F i}-Y_{I i}\right) \sum_{j=1}^{13} a_{j i}\left(\frac{t-t_{I}}{t_{F}-t_{I}}\right)^{j},
$$

where the coefficients $a_{j i}, j=1 \ldots 13$ and $i=1 \ldots 3$, are computed by solving linear equations, whose entries are combinations of the initial and final conditions. In particular, motion planning between two different equilibria $\bar{Y}_{I}$ and $\bar{Y}_{F}$ can be obtained simply by setting $Y_{I}=\bar{Y}_{I}, \dot{Y}_{I}=\ddot{Y}_{I}=\ldots=Y_{I}^{(5)}=$ $Y_{I}^{(6)}=0$ and $Y_{F}=\bar{Y}_{F}, \dot{Y}_{F}=\ddot{Y}_{F}=\ldots=Y_{F}^{(5)}=Y_{F}^{(6)}=0$. The input to be applied that results in the above trajectories is then computed using the flatness property as described in the previous subsection.

\section{Output Feedback Regulation}

We wish to stabilize the crane at a given equilibrium point of the load $\left(\bar{x}_{1}, \bar{x}_{2}, \bar{x}_{3}\right)$ and at a given height of the mobile pulley $\bar{x}_{03}$.

Using the constraints and the dynamic equations at equilibrium, we find the equilibrium values of the remaining variables: $\bar{x}_{11}, \bar{x}_{12}, \bar{L}_{0}, \bar{L}_{1}, \bar{L}_{2}, \bar{L}_{3}$ and the corresponding input torques to be applied: $\bar{T}_{1}, \bar{T}_{2}, \bar{T}_{3}$ and $\bar{T}_{4}$. (Observe that $\bar{T}_{4}=0$ for all equilibria). Define the error variables as $e_{q_{i}}=\bar{q}_{i}-q_{i}$ where $q_{i}$ stands for $i$ th component of $q$. Additionally define $\xi=\arctan \left(\frac{x_{12}}{x_{11}}\right)$, the rotation angle of the rotate platform. Then the corresponding error variable is $e_{\xi}=\bar{\xi}-\xi$.

Recall that the measured variables are: $L_{1}, L_{2}, L_{3}$ and $\xi$. 
Theorem 2. The four PD controllers,

$$
\begin{aligned}
& T_{1}=\bar{T}_{1}+k_{d 1} \dot{e}_{L_{1}}+k_{p 1} e_{L_{1}} \\
& T_{2}=\bar{T}_{2}+k_{d 2} \dot{e}_{L_{2}}+k_{p 2} e_{L_{2}} \\
& T_{3}=\bar{T}_{3}+k_{d 3} \dot{e}_{L_{3}}+k_{p 3} e_{L_{3}} \\
& T_{4}=k_{d 4} \dot{e}_{\xi}+k_{p 4} e_{\xi},
\end{aligned}
$$

applied to the crane dynamics (1) with Constraints (2) assure closed-loop global stability of the equilibrium $\left(\bar{x}_{1}, \bar{x}_{2}, \bar{x}_{3}, \bar{x}_{03}\right)$.

The proof relies on two lemmas as in [11]. Let us define the following energylike function:

$$
W=W_{k}+W_{p}+W_{c t r l},
$$

with

$$
W_{c t r l}=\frac{1}{2}\left(\sum_{i=1}^{3} k_{p i} e_{L_{i}}^{2}+k_{p 4} e_{\xi}^{2}\right)+\sum_{i=1}^{3} \bar{T}_{i} e_{L_{i}}+\bar{T}_{4} e_{\xi}
$$

the "potential" energy stored in the controllers.

Lemma 1. The derivative of $W$ along closed-loop trajectories is given by:

$$
\dot{W}=-k_{d 1} \dot{e}_{L_{1}}^{2}-k_{d 2} \dot{e}_{L_{2}}^{2}-k_{d 3} \dot{e}_{L_{3}}^{2}-k_{d 4} \dot{e}_{\xi}^{2} .
$$

Lemma 2. The only invariant trajectory compatible with $\dot{W}=0$ is the equilibrium trajectory, i.e. $x_{1}(t) \equiv \bar{x}_{1}, x_{2}(t) \equiv \bar{x}_{2}, x_{3}(t) \equiv \bar{x}_{3}$ and $x_{03}(t)=\bar{x}_{03}$.

\section{Extension to Tracking}

Assume that a reference trajectory is constructed so as to steer the load from an idle point to another idle point with obstacle avoidance. This can be done using polynomials as described in Section 4 . Denote the polynomial reference trajectory of the flat output by $Y_{c}$.

Based on flatness, one can calculate the reference trajectory of all other variables in the system as functions of $Y_{c}, \dot{Y}_{c}, \ddot{Y}_{c}, \ldots, Y_{c}^{(6)}$. Denote by $q_{c}=$ $\left(q_{1 c} \ldots q_{12 c}\right)^{T}=\left(x_{1 c}, x_{2 c}, x_{3 c}, x_{01 c}, x_{02 c}, x_{03 c}, x_{11 c}, x_{12 c}, L_{0 c}, L_{1 c}, L_{2 c}, L_{3 c}\right)^{T}$ the reference trajectory of all system variables and by $T_{1 c}, T_{2 c}, T_{3 c}, T_{4 c}$ the reference inputs.

We investigate in this section the closed-loop behavior of the system using the same PD regulator as before but fed by the above references. This modified controller is referred to as the tracking controller. 
Define $e_{q_{i}}=q_{i c}-q_{i}$ where $q_{i}$ is the $i$ th component of the vector $q$ and $q_{i c}$ is the $i$ th component of $q_{c}$. The tracking PD controller is given by:

$$
\begin{aligned}
& T_{1}=T_{1 c}+k_{d 1} \dot{e}_{L_{1}}+k_{p 1} e_{L_{1}} \\
& T_{2}=T_{2 c}+k_{d 2} \dot{e}_{L_{2}}+k_{p 2} e_{L_{2}} \\
& T_{3}=T_{3 c}+k_{d 3} \dot{e}_{L_{3}}+k_{p 3} e_{L_{3}} \\
& T_{4}=T_{4 c}+k_{d 4} \dot{e}_{\xi}+k_{p 4} e_{\xi} .
\end{aligned}
$$

Note that for equilibrium trajectories we get the same $\mathrm{PD}$ regulator as before.

Theorem 3. Let the final point $q_{c}\left(t_{F}\right)$ of the reference trajectory be an equilibrium of the system and suppose that all derivatives along the reference trajectory are bounded. Then $q_{c}\left(t_{F}\right)$ is asymptotically stable in closed-loop using the tracking $P D$ controller.

The stabilization property of the tracking controller given by (8) has been validated using simulation. Comparison of the closed-loop behavior of the two controllers during point to point steering is undertaken. The global stabilizing controller is fed by the equilibrium reference of the desired final point and the tracking controller is fed by the reference trajectory obtained by flatnessbased motion planning.

Two reference trajectories connecting the same initial and final points with transit time of 2.5 seconds are envisaged. The first trajectory (Figures $3-6$ ) is a horizontal displacement. The second one (Figures 7-9) is a parabola in the vertical plane determined by the two points. The globally stabilizing controller produces the same behavior in both cases with damped oscillations, while the tracking controller stabilizes the desired reference and arrives at the equilibrium faster and with less oscillations. The same gains $k_{d i}, k_{p i}$ $(i=1 \ldots 4)$ are used for both controllers.

Notice that the tracking controller outperforms the global one, hence decreasing both the residual sway and the reaching time.

\section{References}

1. G. Corriga, A. Giua, and G. Usai. An implicit gain-scheduling controller for cranes. IEEE Transactions on Control Systems Technology, 6(1):15-20, January 1998.

2. B. D'Andréa and J. Lévine. Modelling and nonlinear control of an overhead crane. In J.H. van Schuppen M.A. Kashoek and A.C.M. Ran, editors, Progress in Systems and Control Theory 4, Robust Control of Linear Systems and Nonlinear Control, Proc. MTNS'91, Vol. II, pages 523-529. Birkhäuser, 1990.

3. M. Fliess, J. Lévine, Ph. Martin, and P. Rouchon. Linéarisation par bouclage dynamique et transformations de Lie-Bäcklund. C.R. Acad. Sci. Paris, I317:981-986, 1993. 

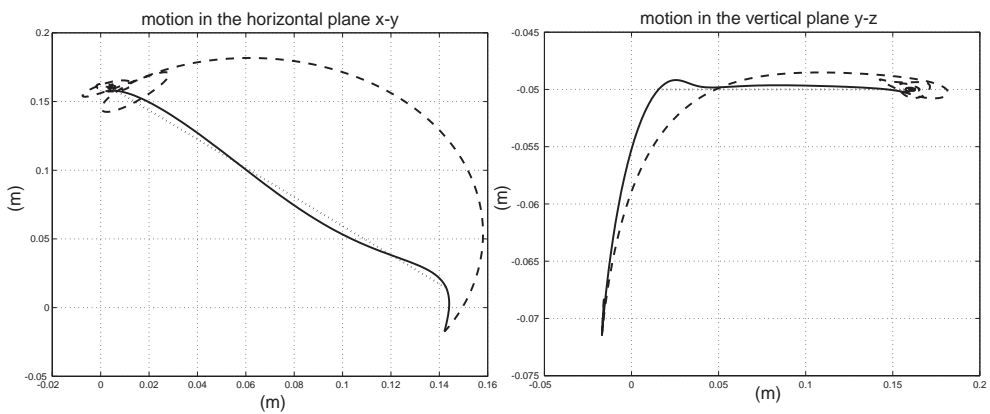

Fig. 3. Closed-loop tracking behavior under PD control. Trajectories of the load in the horizontal and vertical planes: $i$ ) global stabilizing equilibrium controller (hashed line); ii) tracking controller with motion planning; iii) reference to steer to equilibrium along a straight line (dotted).
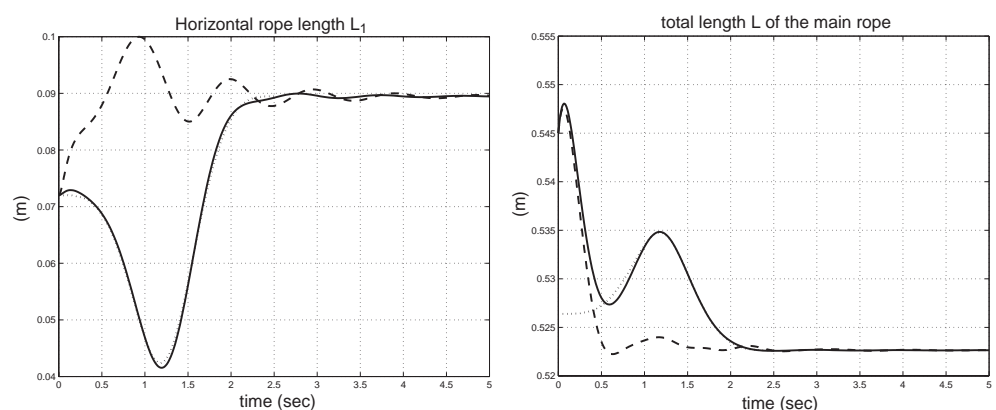

Fig. 4. Closed-loop tracking behavior, rope lengths: $i$ ) global stabilizing equilibrium controller (hashed line); $i$ i) tracking controller with motion planning; iii) reference to steer to equilibrium along a straight line (dotted).

4. M. Fliess, J. Lévine, Ph. Martin, and P. Rouchon. Flatness and defect of nonlinear systems: introductory theory and examples. International Journal of Control, 61(6):1327-1361, 1995.

5. M. Fliess, J. Lévine, Ph. Martin, and P. Rouchon. A Lie-Bäcklund approach to equivalence and flatness of nonlinear systems. IEEE Transactions on Automatic Control, 38:700-716, 1999.

6. M. Fliess, J. Lévine, and P. Rouchon. A generalised state variable representation for a simplified crane description. International Journal of Control, 58:277-283, 1993.

7. D. Fragopoulos, M.P. Spathopoulos, and Y. Zheng. A pendulation control system for offshore lifting operations. In Proceedings of the 14 th IFAC Triennial World Congress, pages 465-470, Beijing, P.R. China, 1999.

8. T. Gustafsson. On the design and implementation of a rotary crane controller. European Journal of Control, 2(3):166-175, March 1996.

9. K.S Hong, J.H. Kim, and K.I. Lee. Control of a container crane: Fast traversing, and residual sway control from the perspective of controlling an underactuated 

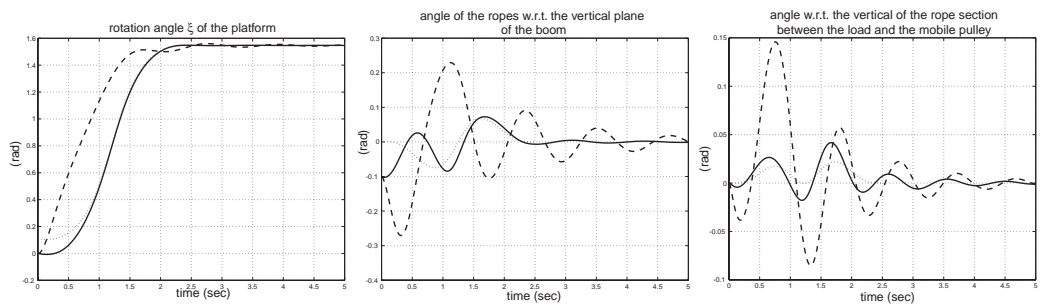

Fig. 5. Closed-loop tracking behavior, angles: $i$ ) global stabilizing equilibrium controller (hashed line); ii) tracking controller with motion planning; iii) reference to steer to equilibrium along a straight line (dotted).
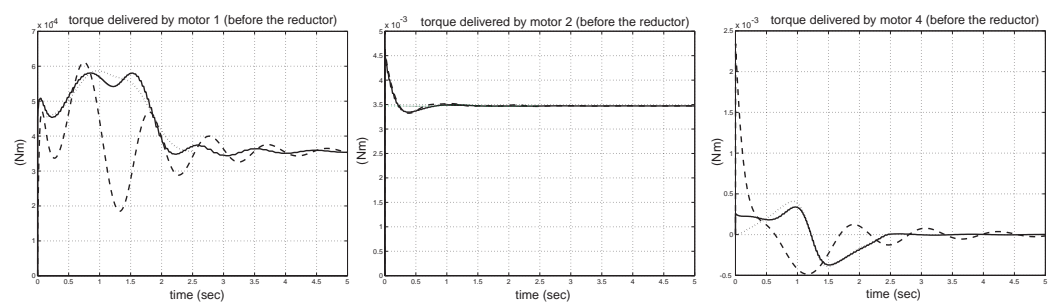

Fig. 6. Closed-loop tracking behavior, motor tensions: $i$ ) global stabilizing equilibrium controller (hashed line); ii) tracking controller with motion planning; iii) reference to steer to equilibrium along a straight line (dotted).

system. In Proceedings of the ACC, pages 1294-1298, Philadelphia, PA, June 1998.

10. B Kiss, J. Lévine, and $\mathrm{Ph}$. Mullhaupt. Modeling and motion planning for a class of weight handling equipments. In Proceedings of the 14th International Conference on Systems Engineering, Coventry, UK, September 2000.

11. B Kiss, J. Lévine, and Ph. Mullhaupt. A simple output feedback PD controller for nonlinear cranes. submitted to: 39th IEEE Conference on Decision and Control, Sydney, Australia, December 2000.

12. J. La Salle and S. Lefschetz. Stability by Liapunov's Direct Method With Applications. Mathematics in Science and Engineering. Academic Press, New York, London, 1961.

13. J. Lévine. Are there new industrial perspectives in the control of mechanical systems ? In Paul M. Frank, editor, Advances in Control, pages 195-226. Springer-Verlag, London, 1999.

14. J. Lévine, P. Rouchon, G. Yuan, C. Grebogi, B.R. Hunt, E. Kostelich, E. Ott, and J. Yorke. On the control of US Navy cranes. In Proceedings of the European Control Conference, pages 213-217, Brussels, Belgium, July 1997.

15. A. Marttinen, J. Virkkunen, and R.T. Salminen. Control study with a pilot crane. IEEE Trans. Edu., 33:298-305, 1990.

16. R.H. Overton. Anti-sway control system for cantilever cranes. United States Patent, (5,526,946), June 1996. 

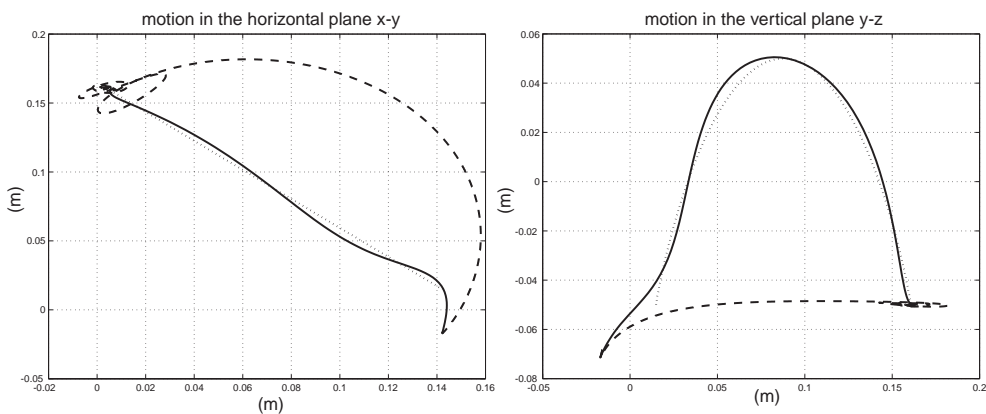

Fig. 7. Closed-loop tracking behavior under PD control. Trajectories of the load in the horizontal and vertical planes: $i$ ) global stabilizing equilibrium controller (hashed line); ii) tracking controller with motion planning; iii) reference to steer to equilibrium along a parabola (dotted).
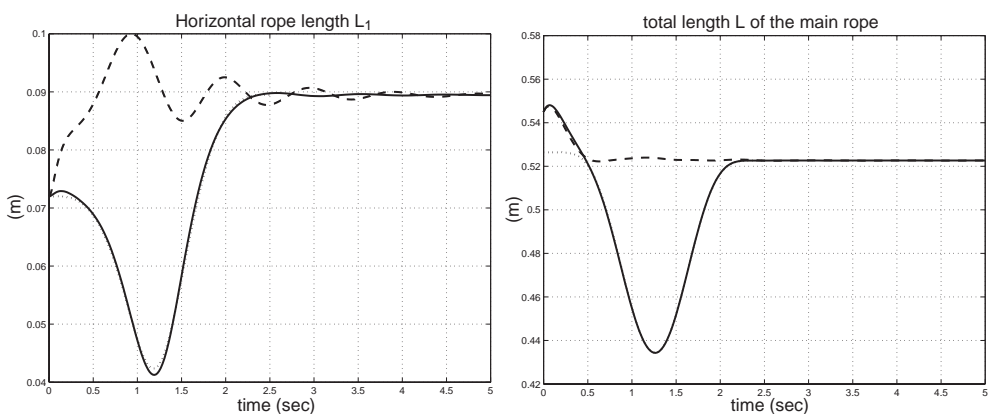

Fig. 8. Closed-loop tracking behavior, rope lengths: $i$ ) global stabilizing equilibrium controller (hashed line); $i$ i) tracking controller with motion planning; iii) reference to steer to equilibrium along a parabola (dotted).
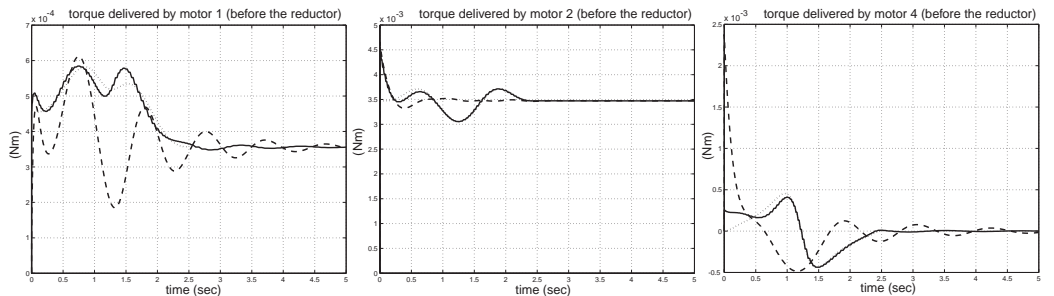

Fig. 9. Closed-loop tracking behavior, motor tensions: $i$ ) global stabilizing equilibrium controller (hashed line); $i$ i) tracking controller with motion planning; iii) reference to steer to equilibrium along a parabola (dotted). 